\title{
On the Measurement of two Independent Viscoelastic Functions with Instrumented Indentation Tests
}

\author{
H. Fadil ${ }^{1}$ - D. Jelagin ${ }^{1} \cdot$ P.-L. Larsson ${ }^{2}$
}

Received: 24 March 2017 / Accepted: 27 September 2017 /Published online: 10 October 2017

(C) The Author(s) 2017. This article is an open access publication

\begin{abstract}
In the present paper, a methodology for complete characterization of linear isotropic viscoelastic material with spherical instrumented indentation test is proposed. The developed method allows for measuring two independent viscoelastic functions, shear relaxation modulus and time-dependent Poisson's ratio, from the indentation test data obtained at non-decreasing loading, but otherwise arbitrary. Finite element modelling (FEM) is relied upon for validating the proposed methodology and for quantifying the influence of experimental variables on the measurements accuracy. Spherical indentation experiments are performed on several viscoelastic materials: polyoxymethylene, bitumen and bitumen-filler mastics. The viscoelastic material functions obtained with the indentation tests are compared with the corresponding results from the standard mechanical tests. Numerical and experimental results presented indicate that the methodology proposed allows mitigating the machine compliance and loading rate effects on the accuracy of the viscoelastic indentation tests.
\end{abstract}

Keywords Indentation - Viscoelasticity $\cdot$ Mechanics of materials $\cdot$ FEM $\cdot$ Bitumen $\cdot$ Bitumen-filler mastics

The original version of this article was revised due to a retrospective Open Access order.

D. Jelagin

denis.jelagin@abe.kth.se

1 Department of Civil and Architectural Engineering, KTH - Royal Institute of Technology, Stockholm, Sweden

2 Department of Solid Mechanics, KTH - Royal Institute of Technology, Stockholm, Sweden

\section{Introduction}

Instrumented indentation tests are used extensively for linear elastic materials characterization in engineering and scientific applications. The major advantages of indentation tests over other characterization techniques are the relative simplicity of the experimental procedure and the possibility to perform characterization at small length scales, as suggested by Oliver and Pharr [1]. The applicability of the indentation tests has further been extended into the domain of non-linear and time-dependent materials, such as viscoelastic materials, e.g. Larsson and Carlsson [2], elastoplastic materials, e.g. Alcalá et al. and Venkatesh et al. [3, 4], and viscoplastic materials, e.g. Bucaille et al. and Storåkers et al. [5, 6]. Considerable attention has also been given in the literature to utilizing the potential of indentation testing to measure the local material properties in inhomogeneous specimens, e.g. Raghavan et al. [7], and Jäger et al. [8].

In the present study, a new experimental and analysis procedure is proposed for indentation testing of linear viscoelastic materials. The proposed procedure allows measuring simultaneously two linearly viscoelastic functions of the material. The primary application of interest here is the viscoelastic characterization of bitumen and bitumen filler mastics. As pointed out in several recent studies, instrumented indentation has certain potential advantages with respect to characterization of bitumenbased materials as compared to other characterization techniques. In particular, indentation testing allows measurement of bitumen's characteristics at micro and nanoscales pertinent to relevant length scales for several modes of damage in asphalt mixtures, e.g. Tarefder and Zaman [9]. Furthermore, bitumen's viscoelastic and adhesive characteristics can be measured simultaneously with indentation testing, Sirghi and Rossi [10]. As also discussed 
by Zofka and Nener-Plante [11] and Jelagin and Larsson [12], indentation is potentially a practical tool to measure spatial variability of viscoelastic parameters in bitumenbased material.

For the case of indentation relaxation test, i.e. when the loading is applied as a Heaviside step function, Larsson and Carlsson [2] proposed, based on the viscoelastic correspondence principle, a simplified solution linking shear relaxation modulus and the measured indentation force history (for the test performed in a displacement control mode). The authors also showed that provided the additional strain field parameter is measured, such as circumferential strain at the specimen surface, one can also determine the bulk relaxation modulus from the test. $\mathrm{H}$. $\mathrm{Lu}$ et al. [13] proposed a methodology to determine shear relaxation modulus based on ramped indentation tests, where the loading is gradually increased during the test. As compared to the simple relaxation test, the ramped indentation scheme requires a more complicated interpretation procedure, since the contact area is not constant. Accordingly, the solution to corresponding contact problem relies upon the functional integrals method originally proposed by Lee and Radok [14]. For the particular case of a ramp followed by creep loading, Oyen [15] developed an analytical formula for obtaining the shear relaxation function. Huang and $\mathrm{Lu}$ [16] expanded the functional integrals technique to determine two independent viscoelastic functions based on the combined indentation tests measurements performed with two different indenter types, one axisymmetric and one non-axisymmetric. In particular, spherical and Berkovich indenters were used in their study.

While the studies discussed above produced results of great interest, a methodology for measuring two independent viscoelastic functions from the indentation data obtained at arbitrary non-decreasing loading history and non-constant Poisson's ratio has not yet been presented. Furthermore, previous experimental studies often report considerable discrepancies between the viscoelastic properties from indentation tests and other testing techniques. The discrepancies have been primarily attributed to the influence of material non-linearities on the measurements $[12,16]$. The influences of far field boundary conditions and of the machine compliance on the measurements have that have discussed by, Hill et al. [17] and Jelagin and Larsson [12] respectively will be investigated using FE.

Accordingly, in the present study, the methodology proposed by Larsson and Carlsson [2] is extended for the case of arbitrary non-decreasing loading history. In order to validate the methodology developed and to investigate the developed technique's sensitivity to the measurement errors, numerical modelling using the finite element method (FEM) is relied upon.
The developed experimental method is used to measure shear relaxation function and viscoelastic Poisson's ratio of polyoxymethylene (POM) and obtained results are compared with values obtained with uniaxial testing. POM was chosen as a relatively stiff material with well-documented viscoelastic properties. Viscoelastic shear relaxation functions are also measured on bitumen and bitumen-filler mastics specimens and compared to those obtained with the dynamic shear rheometer. Based on the modelling and experimental results optimal experimental strategy for characterization of bitumen based materials is proposed.

\section{Theoretical Background and Analysis Procedure}

Larsson and Carlsson [2] proposed an experimental and analysis procedure for full characterization of isotropic linear viscoelastic material with instrumented indentation test performed in relaxation mode. They have shown that two independent viscoelastic functions, i.e. volumetric, $K(t)$, and shear, $G(t)$, relaxation functions, can be determined from the indentation test provided that an additional strain field parameter is measured. Larsson and Carlsson [2] suggested using the circumferential strain measurements at the specimen surface, $\epsilon_{\theta}$, as a feasible strain field parameter. In the present study, the analysis procedure presented by Larsson and Carlsson [2] is generalized for the case of arbitrary loading history with non-decreasing contact area. The geometry of the problem to be analyzed is shown in Fig. 1 along with the coordinate system and relevant parameters.

For the case of frictionless indentation of a rigid spherical indenter into a linear elastic specimen the relationships between indentation force $(\mathrm{P})$, depth $(\mathrm{h})$ and circumferential

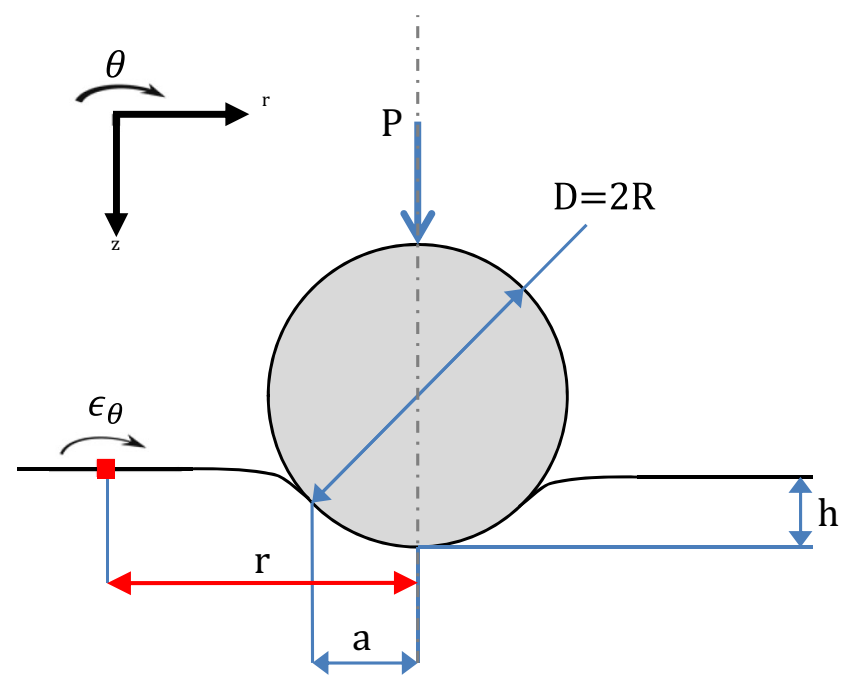

Fig. 1 Sketch of the instrumented indentation test 
strain $\left(\epsilon_{\theta}\right)$ at the surface, are available based on the Hertz theory of elastic contact [18], cf. [19]:

$P=\frac{16}{3} \frac{G a^{3}}{(1-\nu) D}$

$\epsilon_{\theta}(r)=\frac{1}{4} \frac{(2 \nu-1) P}{\pi r^{2} G}$

In equations (1) and (2), $G$ and $v$ are the shear modulus and Poisson's ratio respectively. The radius of the contact area $(a)$ is given by:

$a=\sqrt{h \frac{D}{2}}$

Poisson's ratio can also be expressed as follows:

$\nu=\frac{3 K-2 G}{6 K+2 G}$

where $K$ is the elastic bulk modulus.

By substituting equations (3) and (4) into equations (1) and (2), and utilizing $D=2 R$ the following formulae are obtained after rearranging:

$3 K \times P+4 G \times P=16 \sqrt{R} \times G\left(K \times h^{3 / 2}\right)+\frac{16}{3} \sqrt{R} \times G\left(G \times h^{3 / 2}\right)$

$-3 G \times P=4 \pi r^{2} \times G^{2} \times \epsilon_{\theta}(r)+12 \pi r^{2} \times G \times K \times \epsilon_{\theta}(r)$

However, for the viscoelastic indentation problem with nonconstant contact area, the correspondence principle relied upon by Larsson and Carlsson [2] cannot be applied. Therefore, the method of functional integrals developed by Lee and Radok [14] is used to derive the viscoelastic counterparts of the equations (5) and (6):

$$
\begin{aligned}
& 3 \int_{0}^{t} K(t-\tau) \frac{d P(\tau)}{d \tau} d \tau+4 \int_{0}^{t} G(t-\tau) \frac{d P(\tau)}{d \tau} d \tau \\
& =16 \sqrt{R} \int_{0}^{t} G(t-\tau) \frac{d}{d \tau}\left[\int_{0}^{\tau} K(\tau-\xi) \frac{d h^{3 / 2}(\xi)}{d \xi} d \xi\right] d \tau \\
& +\frac{16}{3} \sqrt{R} \int_{0}^{t} G(t-\tau) \frac{d}{d \tau}\left[\int_{0}^{\tau} G(\tau-\xi) \frac{d h^{3 / 2}(\xi)}{d \xi} d \xi\right] d \tau \\
& -3 \int_{0}^{t} G(t-\tau) \frac{d P(\tau)}{d \tau} d \tau \\
& =4 \pi r^{2} \int_{0}^{t} G(t-\tau) \frac{d}{d \tau}\left[\int_{0}^{\tau} G(\tau-\xi) \frac{d \epsilon_{\theta}(\xi)}{d \xi} d \xi\right] d \tau \\
& +12 \pi r^{2} \int_{0}^{t} G(t-\tau) \frac{d}{d \tau}\left[\int_{0}^{\tau} K(\tau-\xi) \frac{d \epsilon_{\theta}(\xi)}{d \xi} d \xi\right] d \tau
\end{aligned}
$$

Equations (7) and (8) represent the Boltzmann superposition principle applied to the Hertzian solution and the circumferential strain field equation respectively. The first equation represents the relationship between the variation of the indentation load $\delta P$ and the variation of indentation depth $\delta h$ through the relaxation functions $G(t)$ and $K(t)$. The second equation represents the relationship between $\delta P$ and the variation of the circumferential strain $\delta \epsilon_{\theta}$.

To obtain the viscoelastic functions the $P(t), h(t)$, and $\epsilon_{\theta}(t)$ measured during the indentation tests, are used according to the following steps:

- The recorded $P(t), h^{\frac{3}{2}}(t)$ and $\epsilon_{\theta}(t)$, or their slopes, are fitted into functions depending on the loading history and material relaxation behaviour. In the present study, a five terms Prony series is used to fit the load and circumferential functions for ramp or relaxation, while a 5 th degree polynomial was suitable for fitting the experimentally measured indentation depth.

- The viscoelastic functions $G(t)$ and $K(t)$, are subsequently expressed in the Prony series form:

$G(t)=G_{0} \times\left(1-\sum_{i=1}^{n} g_{i} \times\left(1-e^{-t / \tau_{i}}\right)\right)$

$K(t)=K_{0} \times\left(1-\sum_{i=1}^{n} k_{i} \times\left(1-e^{-t / \tau_{i}}\right)\right)$

- The fitted equations $P(t), h^{\frac{3}{2}}(t), \epsilon_{\theta}(t)$ and the Prony series forms of $G(t)$ and $K(t)$ are substituted into equations (7) and (8) and the equations are solved for Prony series parameters: $G_{0}, K_{0}, g_{i}, k_{i}$ and $\tau_{i}$ using a non-linear optimization algorithm.

The need for a non-linear optimization is mitigated provided that the Poisson's ratio is constant and known, as seen in equation (11):

$P(t)=\frac{8}{3(1-\nu)} \sqrt{R} \int_{0}^{t} G(t-\tau) \times \frac{d h^{3 / 2}(\tau)}{d \tau} d \tau$

The problem can now be linearized by assuming the values of time constants in the viscoelastic function of equation (9). The time constants can be assumed as distributed linearly over the time period of interest, the number of Prony series terms is determined by increasing the number of terms gradually until two consecutive analyses give similar solutions.

By substituting $P(t), h^{\frac{3}{2}}(t)$ or their slopes and also $\nu$ or $K(t)$ and integrating equation (11), an equation in the form of equation (12) is obtained. The equation should be satisfied at all 
testing time points and can be solved as an overdetermined system of equations, having the following form:

$f\left(g_{i}\right)=\sum_{i=1}^{n} a_{i} \times g_{i}=0$

where $g_{i}$ are the unknown parameters and $a_{i}$ are constants that arise due to the substitution of $P, h^{3 / 2}$ and the particular time step.

The general solution presented in equations (7), (8) and (11) can also be applied for an indentation test with multiple loading stages, e.g. for a linearly increasing load followed by a constant load.

For the particular case of constant Poisson's ratio, equations (7) and (8) are reduced to the following equation, originally proposed by Oyen [15]:

$$
\begin{aligned}
& P(t)= \frac{8}{3(1-\nu)} \sqrt{R} \int_{0}^{t_{1}} G_{2}(t-\tau) \times \frac{d h_{1}^{3 / 2}(\tau)}{d \tau} d \tau \\
&+\frac{16}{3} \sqrt{R} \int_{t_{1}}^{t} G_{2}(t-\tau) \times \frac{d h_{2}^{3 / 2}(\tau)}{d \tau} d \tau \\
& \text { for } t \geq t 1
\end{aligned}
$$

For the particular loading case of the relaxation indentation test, i.e. $h(t)=h_{o} H(t)$, equations (7)-(8) reduce to the following form in the Laplace transformed domain:

$$
\begin{aligned}
& \tilde{P}=\frac{16}{3} \sqrt{R} \times \frac{s \tilde{G}(3 \tilde{K}+\tilde{G})}{(3 \tilde{K}+4 \tilde{G})} \times \frac{h_{o}^{3 / 2}}{s} \\
& \tilde{\epsilon}_{\theta}(r)=-\frac{3}{4} \frac{\tilde{P}}{\pi r^{2}(3 \tilde{K}+\tilde{G}) \times s}
\end{aligned}
$$

Here $\mathcal{L}\left(h^{3 / 2}\right)=\frac{h_{o}^{3 / 2}}{s}$ has been utilized since $h$ is constant during the test and the tilde $(\sim)$ symbol above the variables denotes the Laplace transform of these parameters.

The viscoelastic functions are obtained by transforming the functions resulting from fitting the measurements of the force and the circumferential strain, and solving equations (14) and (15) simultaneously for the relaxation functions in the Laplace domain $\tilde{G}$ and $\tilde{K}$. The relaxation functions are then transformed into the time domain by taking the inverse Laplace transform of $\tilde{G}$ and $\tilde{K}$ numerically using the Talbot algorithm.

The problem becomes considerably simpler when the Poisson's ratio is constant and known; this can be relevant for materials that are nearly incompressible in practical applications, such as bitumen. In this case equations (14) and (15) are reduced to a single equation in a time domain:

$G(t)=\frac{3}{16} \frac{(1-\nu) D}{a^{3}} P(t)$
Equation (4) was used to replace $K(t)$ with $\nu(t)$. Equations (14)-(16) coincide with the ones originally proposed by Larsson and Carlsson [2].

\section{Computational Study}

In order to validate the developed solutions, a finite element model of the instrumented indentation test on linear viscoelastic material has been developed in the commercial FE software ABAQUS. The spherical indenter was modelled as a rigid body and axial symmetry was utilized for the whole model. The specimen's viscoelastic material behaviour is described by a one term Prony series (Eqs. (9)-(10)), with the following material constants:

$$
\begin{array}{lrr}
G_{0}=83.11 M P a & & K_{0}=176.94 M P a \\
g_{1}=0.8 & k_{1}=0.6 & \tau_{1}=0.6 s
\end{array}
$$

The shear relaxation modulus constants are derived from a Prony series with one term fitted for the indentation results obtained by Jelagin and Larsson [12]. However, the bulk relaxation modulus constants were chosen to follow the same shape as the shear relaxation modulus, and to result in a constant Poisson's ration of 0.3 .

The FE mesh used is shown in Fig. 2. As seen in Fig. 2 the mesh was divided into three regions with varying element sizes, with the higher elements density close to the contact region. The bottom part of the specimen is assumed to be fixed in the vertical direction. The elements used had 8-nodes and were two dimensional axisymmetric elements.

In order to validate the analysis procedures indentation tests were modelled for two different types of loading history. Namely, the indentation ramp-relaxation test, where the indentation depth was monotonically increased from zero to a target maximum value, $h_{f}$, within time of $t_{r}$, and the indentation relaxation test, where the material was first loaded elastically to a target depth, $\mathrm{h}_{\mathrm{f}}$, followed by a viscoelastic relaxation step at constant $h$. Equation (18) shows the

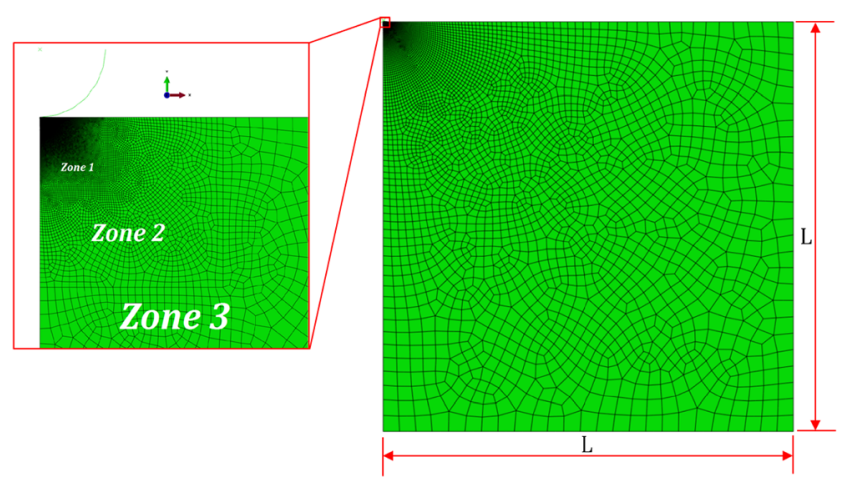

Fig. 2 The indentation specimen dimensions and a zoomed-in view of the mesh and the different zones used 
relaxation indentation test, while equation (19) describes the ramp-relaxation indentation test:

$h(t)=h_{f} \times H(t)$

$h(t)= \begin{cases}\frac{t}{t_{r}} h_{f} & \text { for } t \leq t_{r} \\ h f & \text { for } t \geq t_{r}\end{cases}$

where $H(t)$ is the Heaviside function. The test performed with the loading according to equation (19), but only for $t \leq t_{r}$, the test is referred to as a ramp indentation test below.

$P(t), h(t)$ and $\epsilon_{\theta}(t)$ obtained computationally were used to back-calculate the specimens viscoelastic parameters, $G(t)$ and $\nu(t)$. For the model of ramp and ramp-relaxation indentation tests the analysis procedure based on equations (7) and (8) was used. For the relaxation test the procedure originally proposed by Larsson and Carlsson [2], i.e. equations (14) and (15), was relied upon.

The FE model was also used to investigate the potential sources of measurement errors in indentation tests and to quantify their effect on the measured $G(t)$ and $\nu(t)$. In particular, the influence of the finite specimen size on the measured viscoelastic functions was examined with comparative simulations performed for the range of relative specimen sizes, $L / a$, $L$ being distance from the specimens symmetry axis to its vertical and horizontal boundaries. As it is also known, measurement of $h(t)$ and $\epsilon_{\theta}(t)$ is prone to errors due to difficulties associated with identification of the point of first contact, cf. VanLandingham et al. [20] and limited accuracy of strain gages respectively. Accordingly, the influence of the measurement errors in $h(t)$ and $\epsilon_{\theta}(t)$, on the resulting $G(t)$ and $\nu(t)$ has been examined numerically.

One limitation of the indentation relaxation tests is that it is impossible to enforce exactly the target indentation depth history; i.e. equation (18). Obviously, no device is capable of applying infinitely fast loading rate. Also, as illustrated by Jelagin and Larsson [12], machine compliance has to be taken into account for obtaining the correct viscoelastic material properties. In order to obtain some quantitative insight regarding the influence of the loading history on the measured viscoelastic parameters, simulations of relaxation tests were performed for a range of loading rates. Machine compliance effect, has furthermore been modelled by replacing the constant indentation depth during the relaxation part of the test with a power law function to simulate the effect observed by Jelagin and Larsson [12].

\section{Experimental Study}

Spherical indentation tests were performed on polyoxymethylene (POM) and on a range of bitumen-based materials; a spherical steel indenter, with $8 \mathrm{~mm}$ curvature radius was used in all tests, the effect of the indenter curvature radius on the shear relaxation function measurement was examined in [12]. POM is a relatively stiff thermoplastic material, with $\nu(t)<0.5$, at room temperature. Accordingly, indentation tests on POM were used to evaluate the capability of the analysis procedures based on equations (7) and (8) and (14) and (15) to extract two independent viscoelastic functions from the indentation test data. Bitumen, on the contrary, has $\nu(t)$ very close to 0.5 , at least at the temperatures above the glass transition temperature. Correspondingly, bitumen-based specimens are assumed, for simplification, to be completely incompressible in the present study. Only the viscoelastic shear relaxation modulus $G(t)$ has been measured. Indentation tests were performed on neat bitumen as well as on bitumen-filler mastics specimens.

The bitumen used in the present investigation is unmodified 70-100 penetration grade bitumen provided by Nynas AB (Nynashamn, Sweden). Results of the basic physical and chemical characterization of the bitumen used are summarized in Table 1. For a detailed description of the tests mentioned in Table 1 the reader is referred to the corresponding standards. Bitumen-filler mastics specimens were prepared by mixing bitumen with $10 \%$ and $20 \%$ by weight of hydrated lime filler. The mastics were prepared by mixing hydrated lime and bitumen in a container at $150{ }^{\circ} \mathrm{C}$. After adding pre-weighed hydrated lime filler to $70 \mathrm{~g}$ bitumen, the mixtures were stirred at $600 \mathrm{rpm}$ for about $10 \mathrm{~min}$.

In order to verify the indentation test measurements, the viscoelastic properties of the materials investigated were
Table 1 Physical and chemical properties of bitumen, [21]

\begin{tabular}{|c|c|c|}
\hline Test & Value & Standard \\
\hline Penetration & $8.6 \mathrm{~mm}$ & EN 1426 \\
\hline Softening point & $46.4^{\circ} \mathrm{C}$ & EN 1427 \\
\hline Dynamic viscosity $60^{\circ} \mathrm{C}$ & 96 Pa.s & EN 12596 \\
\hline Fraass breaking point & $-16{ }^{\circ} \mathrm{C}$ & EN 12593 \\
\hline Bending beam rheometer at $-15^{\circ} \mathrm{C}$ & $\begin{array}{l}\text { stiffness }=143 \mathrm{MPa}, \mathrm{m}=0.37 \\
\quad(\text { loading time } 60 \mathrm{~s})\end{array}$ & EN 14771 \\
\hline Bending beam rheometer at $-25^{\circ} \mathrm{C}$ & $\begin{array}{l}\text { stiffness }=524 \mathrm{MPa}, \mathrm{m}=0.26 \\
\quad \text { (loading time 60s) }\end{array}$ & EN 14771 \\
\hline Wax content by differential scanning calorimetry & $6.2 \%$ & According to $[21]$ \\
\hline
\end{tabular}


also measured with the uniaxial tension test and the dynamic shear rheometer (DSR), for POM and bitumenbased materials respectively.

\section{Linear Viscoelastic Characterization of POM}

The uniaxial tensile test setup is shown in Fig. 3 along with specimens' shape and dimensions. An INSTRON servohydraulic load frame with a $2 \mathrm{kN}$ load cell was used for the tests, with an accuracy of $0.5 \%$ of the measured load, in the range $10 \mathrm{~N}$ to $2 \mathrm{kN}$. An extensometer with gauge length of $12.5 \mathrm{~mm}$ was used to measure specimens' axial strain. In order to measure the materials Poisson's ratio, the specimens' circumferential strain was measured with a $5 \mathrm{~mm}$ strain gauge glued around the specimen circumference as shown in Fig. 3(a). Three POM specimens have been prepared for the uniaxial tests, with their shape and dimensions as shown Fig. 3(a). The specimens' diameter, $d_{u}$, was approximately $5 \mathrm{~mm}$ with $\pm 0.03 \mathrm{~mm}$ variations between specimens. For each specimen, the smallest diameter was measured using a digital caliper and the measured value was used to calculate specimens' crosssectional area.

The specimens were tested in uniaxial creep, at room temperature, by assigning the constant loads of 400, 300 and $200 \mathrm{~N}$ that correspond approximately to the stress levels of 20, 15 and $10 \mathrm{MPa}$. According to the experimental results by Starkova and Aniskevich [22], POM remains in the linear viscoelastic range for the stress levels below $20 \mathrm{MPa}$. The load was increased monotonically to a target level in less than $0.9 \mathrm{~s}$ and then it was maintained constant for $15 \mathrm{~min}$. Two tests per specimen and load level have been performed resulting in 18 measurements total. The specimens were left to recover for $25 \mathrm{~min}$ between the tests, the duration of the recovery period has been determined as the time required for the extensometer to record zero strains. In the uniaxial tensile test, $\nu(t)$ was calculated from the measured circumferential and longitudinal strains, $\epsilon_{\theta}(\mathrm{t})$ and $\epsilon_{\mathrm{z}}(\mathrm{t})$, as:

$\nu(t)=-\frac{\epsilon_{\theta}(t)}{\epsilon_{z}(t)}$

For the instrumented indentation tests a cubic POM sample with dimensions of $7 \times 7 \times 7 \mathrm{~cm}^{3}$ was used. In order to reduce the influence of surface roughness on the measurements, the specimens surface was first grounded with fine sandpaper and then polished with ISO P1200 sandpaper (grain size $15.3 \pm 1.0 \mu \mathrm{m})$.

A hydraulic load frame used for the indentation tests is shown in Fig. 4. A $50 \mathrm{kN}$ load cell with an accuracy of $0.5 \%$ of the measured value was used.

The indentation depth was measured by using the position of the piston, within a resolution of $\pm 0.5 \mu \mathrm{m}$. The measured machine compliance $C_{m}$ for the test set-up configuration is $0.022 \mu \mathrm{m} / \mathrm{N}$. To calculate the actual indentation depth, a linear machine compliance was assumed; thus, the indentation depth was taken as the difference between the indentation depth as measured by the piston movement and the deformation in the machine at the measured load level. A direct measurement of $h(t)$ would provide more reliable results, it would also allow to at least partially mitigate problems associated with machine compliance. However, a direct measurement of $h(t)$ was not possible with the available laboratory equipment.

In order to measure $\epsilon_{\theta}(t)$ induced at specimen's surface during the indentation test a half-bridge strain measurement arrangement with two $5 \mathrm{~mm}$ active strain gauges was used, as shown in Fig. 4. It should be noted that the foil strain gauges would average the strain over the $5 \mathrm{~mm}$ measurement distance, this is a limitation of the strain measurement method used in this study; as it will be assumed that this average strain

Fig. 3 The uniaxial creep test (a) sample with strain gauge attached, (b) Sample mounted on the load frame

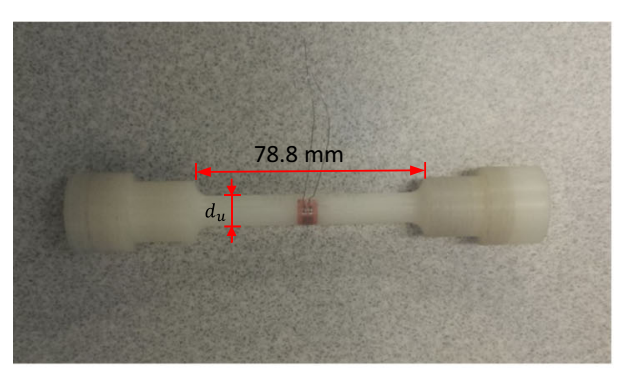

(a)

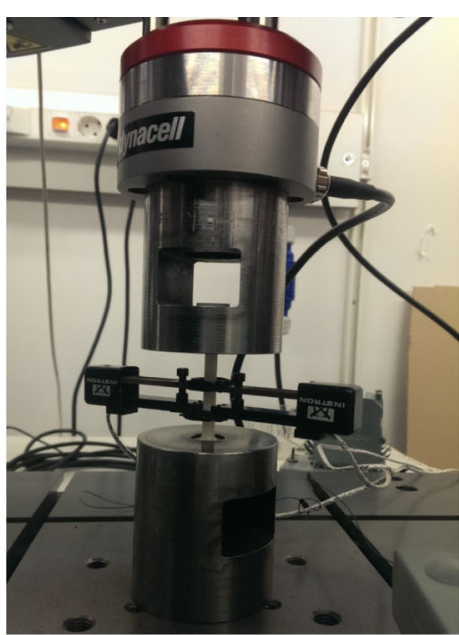

(b) 


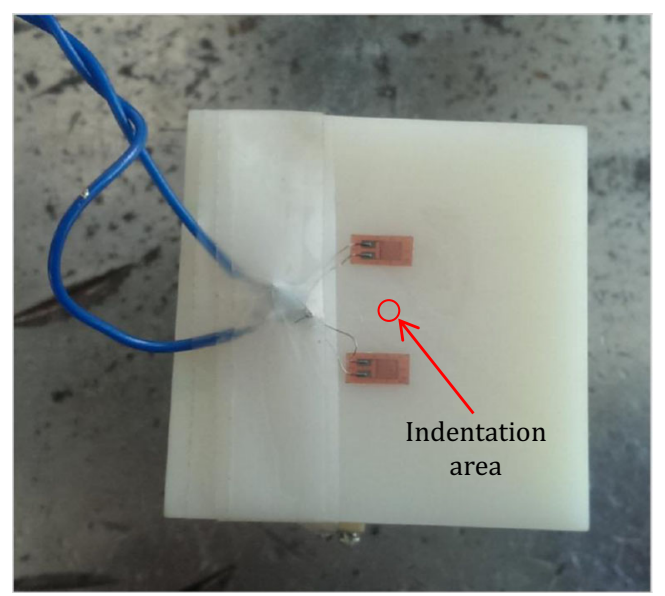

Fig. 4 Area of indentation and strain gauges arrangement on the POM sample

is the local strain in the middle of the gauge. The indentation test was done in the area between the two strain gages (marked with a red circle in Fig. 4). The strain gauges were aligned circumferentially in a half bridge setup. In order to determine the relative position of the strain gages with respect to the indentation location, the steel ball was painted using ink and after the test, the distances were measured using an optical microscope, with an accuracy of at least $15 \mu \mathrm{m}$. The average of the distances measured was used as the sensor location, $r$, shown in Fig. 1. The strain gauges were placed at a distance $r=6.6 \mathrm{~mm}$ from the center of indentation. The distance was chosen so that the strain gauges would be far enough away from the indenter to mitigate the risk of the indenter touching the strain gauge, and yet close enough to have a reasonable amount of strain recorded. A possible alternative to the foil strain gauges would have been strain measurement with a digital image correlation, through this technique the strain field around the indenter can be captured, and the effect of strains measured at different distances from the indenter can be investigated. This would allow mitigating uncertainties associated with strain averaging across the gauge width and would provide more strain measurement points, thus improving the reliability of the technique.

A total of 20 indentation relaxation tests were performed with $h_{f} \leq 70 \mu \mathrm{m}$. In all tests the sampling frequency of $100 \mathrm{~Hz}$, thus the loading time was taken as $0.01 \mathrm{~s}$. The position of the piston was kept constant for $10 \mathrm{~min}$ and the indentation load and specimens' circumferential strain were monitored for the duration of the test. The loading history for the indentation relaxation tests is given by equation (18), which can be modified to take the machine compliance into consideration:

$h(t)=h_{f} \times H(t)-P(t) * C_{m}$

It is worth noting that equations (18) and (21) assume that the machine's loading rate is negligible.
For the ramped indentation tests, the following loading scheme was used. In order to reduce the effect of surface unevenness in the measurements the specimen was first preindented to a prescribed initial piston position $h_{i}$ in one second and the material was allowed to relax for $600 \mathrm{~s}$; then the piston's position was changed monotonically from $h_{i}$ to a final prescribed piston position $h_{f}$ for another $600 \mathrm{~s}$. A total of ten tests were performed. The loading function is given by:

$h(t)= \begin{cases}t \times h_{i}-P(t) * C_{m} & t \leq 1 s \\ h_{i}-P(t) * C_{m} & 1 s \leq t \leq 601 s \\ h_{i}+\frac{(t-601)}{t_{r}} \times\left(h_{f}-h_{i}\right)-P(t) * C_{m} & t \geq 601 s\end{cases}$

\section{Linear Viscoelastic Characterization of Bitumen and Bitumen-Filler Mastics}

Shear relaxation modulus of bitumen and of bitumen-filler mastic specimens were measured with DSR at Nynas AB (Nynashamn, Sweden). All the tests were performed according to EN-14770 at 00C. The limits of linear viscoelasticity for the materials tested have been determined based on the strain sweep tests. The results of the strain sweep tests indicated that both bitumen and bitumen filler mastics specimens exhibited linear behaviour for shear strains below approximately $2 \%$. Accordingly, relaxation and complex modulus tests have been performed on bituminous materials at $\gamma_{\max }=2 \%$ as follows.

In the shear relaxation tests, specimens were loaded in the DSR to $2 \%$ shear strain within approximately $0.04 \mathrm{~s}$; the strain was then maintained constant for at least $50 \mathrm{~s}$, and the resulting shear stress was recorded. The shear relaxation function was obtained then as the ratio of the shear strain to the shear stress. The $G(t)$ for bituminous materials has also been measured with the frequency sweep tests, performed at the frequency range from 0.1 to $100 \mathrm{rad} / \mathrm{s}$ at $0{ }^{\circ} \mathrm{C}$. The complex modulus of the materials, obtained from the tests, was then converted to the viscoelastic shear relaxation function using the method described by Mun et al. [23].

Instrumented indentation tests on bituminous materials have been performed with a set-up shown in Fig. 4. A servo-hydraulic MTS load frame was used with a $10 \mathrm{~N}$ capacity load cell having an accuracy of $0.5 \%$ of the read-out value. Indentation depth was monitored using LVDT sensor with $0.5 \mu \mathrm{m}$ resolution. The tests were performed at $0{ }^{\circ} \mathrm{C}$. A custom-made temperature chamber was used to control the testing temperature. The temperature was monitored throughout the tests with a thermocouple embedded approximately halfway through the thickness of the specimen. The temperature was found not to vary more than $\pm 1{ }^{\circ} \mathrm{C}$ during the tests. The specimens were glued to a heavy metallic block to 
prevent specimen wobbling during the tests. The setup compliance was measured to be $C_{m}=33.68 \mu \mathrm{m} / \mathrm{N}$.

Bitumen and bitumen-filler mastics specimens were poured into cylindrical aluminum containers of $6 \mathrm{~cm}$ diameter. The containers were filled with material up to approximately $2.5 \mathrm{~cm}$ in height (Fig. 5).

Two sets of indentation tests were performed: relaxation indentation tests, and ramp-relaxation indentation tests. In the relaxation indentation tests; the specimens were indented to $h_{f} \leq 70 \mu \mathrm{m}$ with the indentation depth history defined by equation (21). Five tests per material have been performed.

The indentation ramp-relaxation tests were performed with a loading scheme described as follows: the specimen was first pre-indented to a prescribed initial piston position $h_{i}=0.04$ microns in one second and the material was allowed to relax for $50 \mathrm{~s}$; then the piston position was increased monotonically from $h_{i}$ to $h_{f}=0.08$ microns in $1 \mathrm{~s}, h_{f}$ was then kept constant for $50 \mathrm{~s}$. Equation (23) shows the relationship between the actual indentation depth, i.e. the one corrected for machine compliance effect, and the piston position for of the ramp-relaxation indentation tests:

$h(t)= \begin{cases}t \times h_{i}-P(t) * C_{m} & t \leq 1 s \\ h_{i}-P(t) * C_{m} & 1 s \leq t \leq 51 s \\ h_{i}+\frac{(t-51)}{t_{r}} \times\left(h_{f}-h_{i}\right)-P(t) * C_{m} & 51 s \leq t \leq 52 s \\ h_{f}-P(t) * C_{m} & t \geq 52 s\end{cases}$

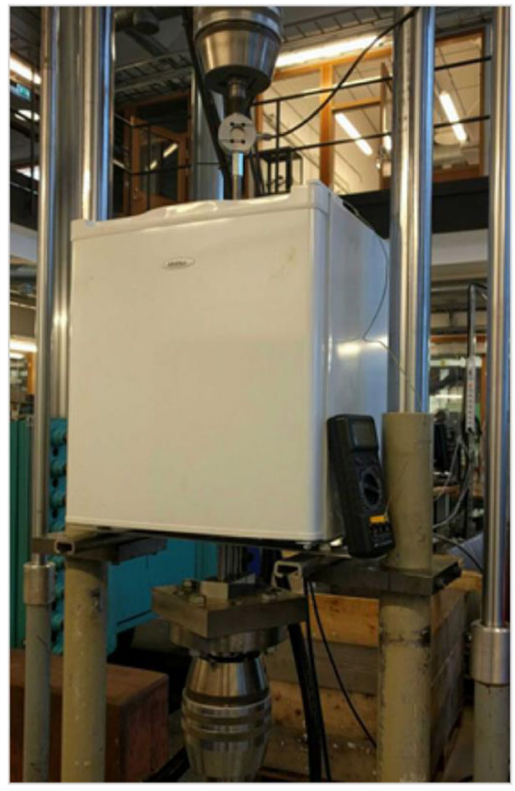

(a)

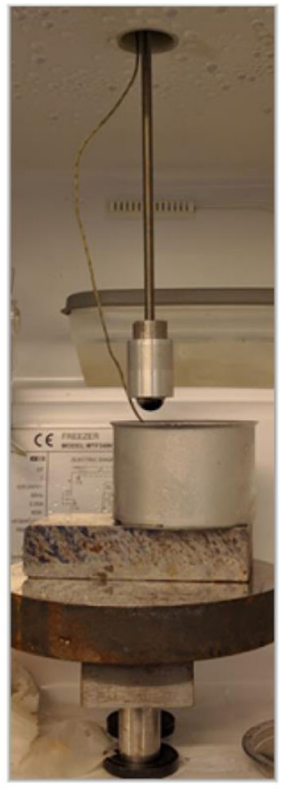

(b)
Fig. 5 The experimental setup for bitumen and mastics test (a) an external view of the temperature chamber $(\mathbf{b})$ an internal view of the temperature chamber showing the indenter and container sample

\section{Results and Discussion}

\section{Computational Study}

The methodology for measuring two independent viscoelastic functions based on the indentation test data obtained at arbitrary non-decreasing loading history has been validated computationally. The analysis procedure based on equations (7-8) was used to back-calculate the $G(t)$ and $\nu(t)$ functions from the $P(t), h(t)$ and $\epsilon_{\theta}(t)$ obtained computationally for the cases of the ramp and ramp-relaxation indentation tests. In Fig. 6 (a), the average errors between input and back-calculated viscoelastic functions is reported as function of relative specimen size $L / a$. Average error reported in Fig. 6(a) are obtained as follows:

$\overline{\mathrm{e}}=\frac{\text { magnitude of error }}{\text { original magnitude }}=\frac{\int_{0}^{10}\left|F_{\text {input }}(t)-F_{\text {obtained }}(t)\right| d t}{\int_{0}^{10}\left|F_{\text {input }}(t)\right| d t}$

where $F_{\text {input }}(t)$ represents the input $G(t)$ and $\nu(t)$, and $F_{\text {obtained }}$ represents the corresponding functions obtained from numerical modelling results. The corresponding input viscoelastic functions are shown in Fig. 6(b) along with $G(t)$ and $\nu(t)$ obtained for the case of $L / a=5$. As may be seen, in Fig. 6, the proposed analysis procedure is able to capture the viscoelastic functions accurately, provided that the effect of finite specimen size is not an issue. As further shown in Fig. 6(a), at least for $L / a>40$, the influence of the finite specimen size on the measurements is small, with input and back-calculated functions deviating less than 1\%. As also seen in Fig. 6(b), $v(t)$ is somewhat more sensitive to the specimen size as compared to $G(t)$. Even for the relative specimen sizes as low as $L / a=10$ the influence on the measured viscoelastic function is quite small with the average difference being within $10 \%$ for both $G(t)$ and $v(t)$. This emphasizes the fact that indentation test measurements are controlled by the local material properties in the vicinity of the contact area.

In Figs. 7 and 8 the ramp and relaxation indentation tests sensitivities to the measurement errors of indentation depth and circumferential strain respectively are investigated. In both figures, the calculated average error in the measured viscoelastic parameters is reported as a function of measurement errors along with the viscoelastic functions, $G(t)$ and $v(t)$, back-calculated from numerical results corresponding to a certain value of measurement error.

One challenge with instrumented indentation testing is the accurate identification of the first contact point, in particular in case of soft materials. In Fig. 7, errors arising in measured $G(t)$ and $v(t)$ due to misidentification of the first contact point are examined based on the computational results. In the FEM simulations, error in the depth measurement was modelled, 


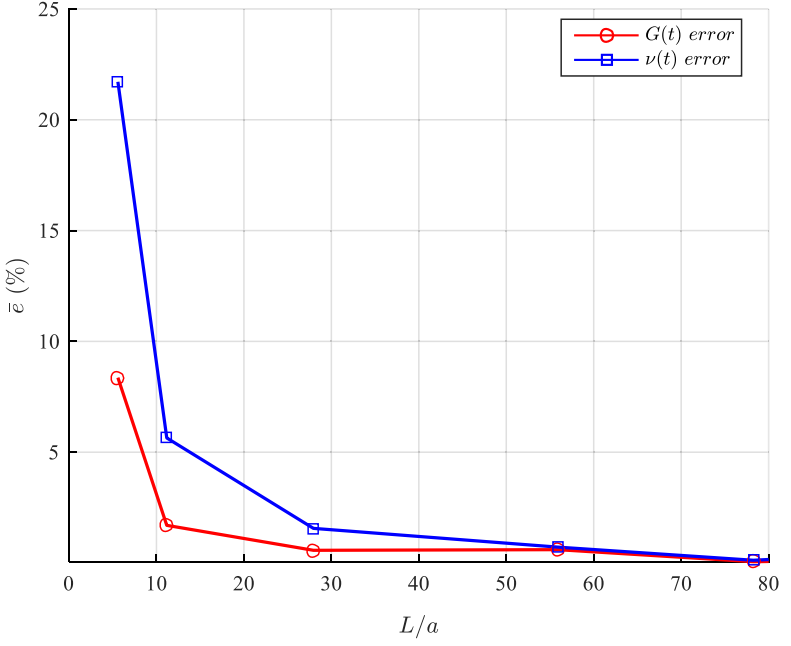

(a)

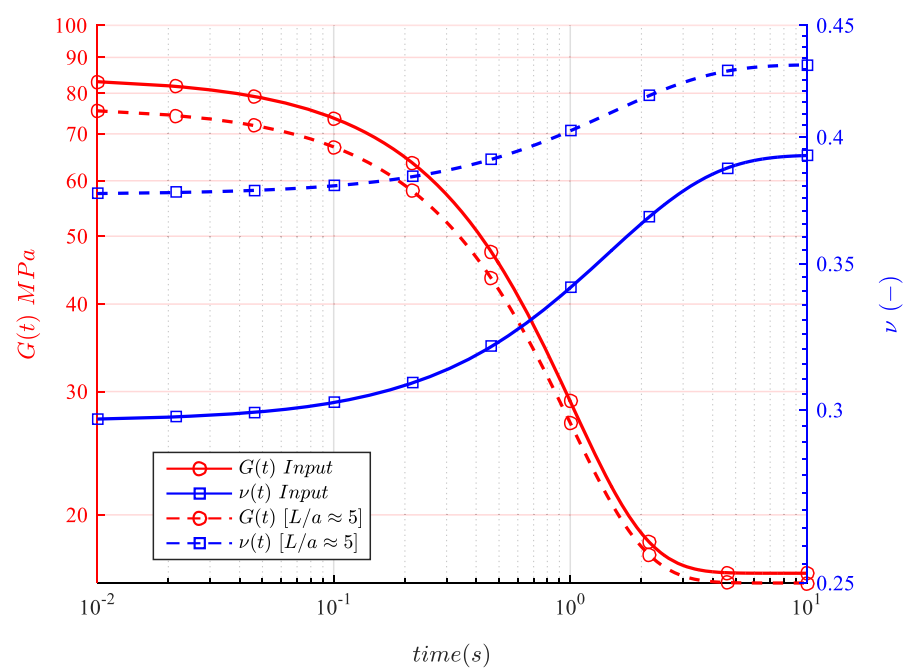

(b)

Fig. 6 (a) The average errors obtained in the calculation of the viscoelastic functions due to specimen size. (b) The input $G(t)$ and $\nu(t)$ vs. the obtained corresponding values for $L / a \approx 5$

as an additional indentation depth, denoted as $h_{e}$, applied to the indenter at the beginning of the test, $t=0$. The relaxation test is described by equation (18), while the ramp test is described by the ramp-relaxation equation (19), for $t \leq t_{r}$. In Fig. 7(a), $E_{\text {average }}$ is reported as a function of $h_{e} / h_{f}$. As may be observed in Fig. 7(a), the relaxation test measurements are somewhat more sensitive to the indentation depth errors as compared to the ramp test; $G(t)$ is also more affected by $h_{e} / h_{f}$ than $v(t)$.

In Fig. 7(b), input $G(t)$ and $v(t)$ are shown along with the ones back-calculated from the model of the ramp test, with $h_{e} / h_{f}=5 \%$. As shown in Fig. 7(b) the effect of misidentification of the first contact point is most profound in the beginning of the test, with approximately $8 \%$ and $6 \%$ error in the measured $G(t=0)$ and $v(t=0)$ correspondingly, and drops to approximately $2 \%$ and $1 \%$ in about $4 \mathrm{~s}$.

The influence of error in circumferential strain measurements on the measured $G(t)$ and $v(t)$ is examined in Fig. 8. The modified circumferential strain, $\epsilon_{\theta}(t)^{\text {mod }}$, is calculated by adding an additional constant error, $\epsilon_{\theta}$ error, to each data point.

In Fig. 8(a) the $E_{\text {average }}$ for the back-calculated viscoelastic functions are presented as a function of $\epsilon_{\theta}$ error. Figure 8(a) shows that $v(t)$ is expectedly more sensitive to the errors in strain measurement. In Fig. 8(b) input and obtained viscoelastic functions are compared for the ramp indentation test showing a 20\% constant error in strain measurement throughout the test. As seen in Fig. 8(b), the 20\% error in strain measurement results in differences between the curves, these differences

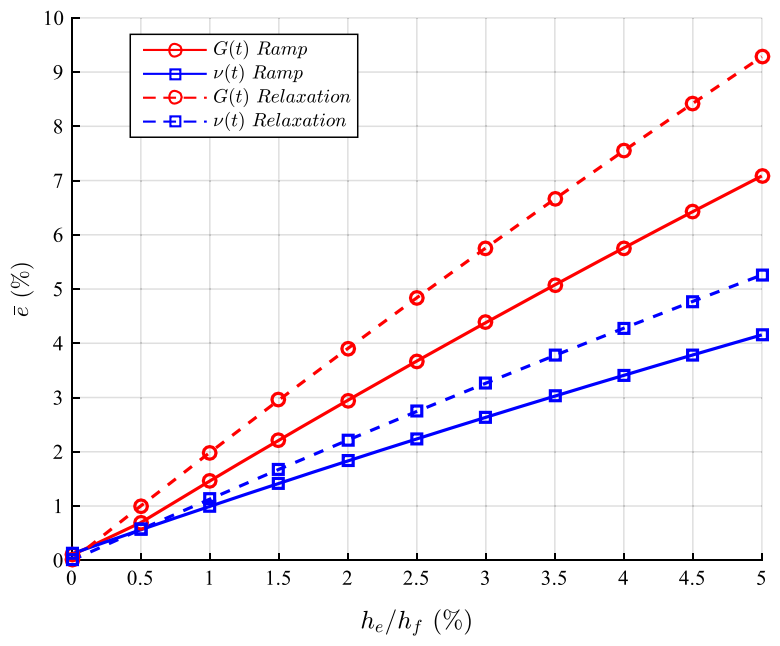

(a)

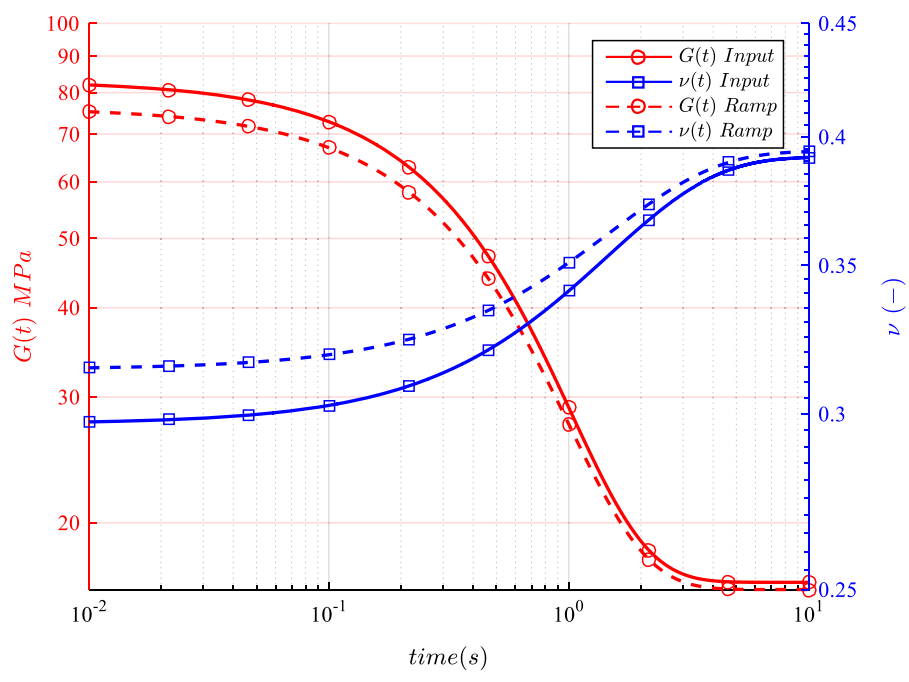

(b)

Fig. 7 (a) The average errors incurred due to the misidentification of the first contact point (b) $G(t)$ and $\nu(t)$ measured for $\frac{h_{e}}{h_{f}}=5 \%$ vs. the input values 


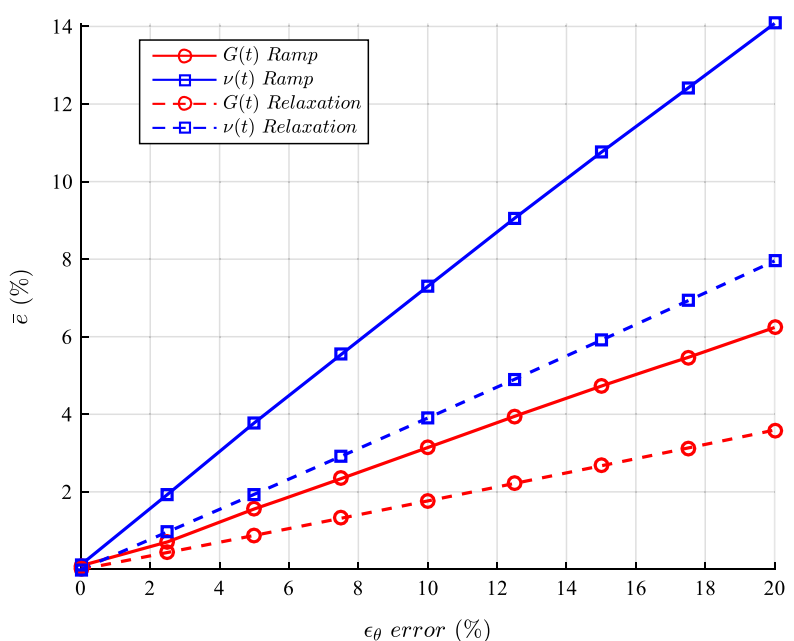

(a)

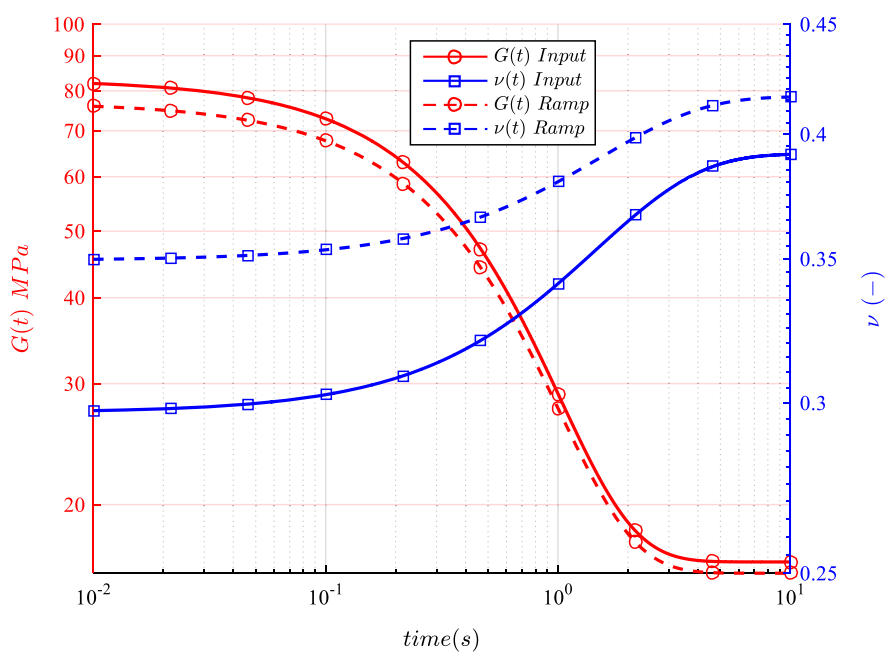

(b)

Fig. 8 (a) The average errors incurred due to the error in $\epsilon_{\theta}(t)$ measuarement (b) $G(t)$ and $\nu(t)$ measured for $\epsilon_{\theta}(t)$ error $=20 \%$ vs. the input values

amount to approximately $18 \%$ and $7 \%$ error in the measured $G(t=0)$ and $v(t=0)$ respectively, and drops to approximately $4 \%$ in $1 \mathrm{~s}$ for $G(t)$ and $6 \%$ in $6 \mathrm{~s}$ for $v(t)$.

As discussed above, enforcing the loading history according to equation (18) for the relaxation indentation test, is not easy. In Fig. 9(a) the influence of finite loading rate in the relaxation test on the measured $G(t)$ is examined. In order to account for the finite loading rate, the simulations have been performed with the loading history according to equation (19), with a range of $t_{r}$ values. The back-calculated $G(t)$ curves are presented as obtained with the analysis procedure for relaxation, i.e. the one based on equations (14) and (15) for a range of finite loading rates. In Fig. 9, $t=0$ corresponds to the start of the relaxation part of the test. Obviously, the effect of finite loading rate on the measured $G(t)$ is dominant in the beginning of the test and deviation between the measured and input curves diminishes fast with time as shown in Fig. 9(a). This is further illustrated in Fig. 9(b) where the relative error between the input and measured curves is presented as a function of time. As seen, for all the cases investigated, the deviation between the measured and input curves is higher than $10 \%$ only for the $t<2.5 \times t_{r}$.

In order to examine the influence of the machine compliance, the indentation tests were modelled for the loading history according to equation (22); with $t_{r}=0.01 \mathrm{~s}$ and $C_{m}=$ $33.68 \mu \mathrm{m} / \mathrm{N}$. This history represents a relaxation test where machine compliance results in indentation depth smaller than the target $h_{f}=0.08 \mathrm{~mm}$ at the start of the relaxation period;

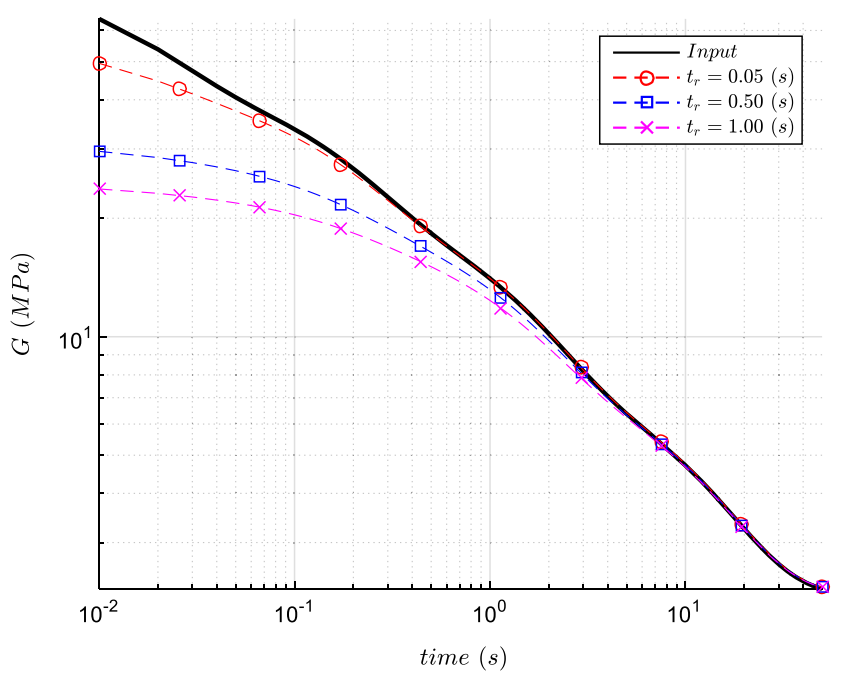

(a)

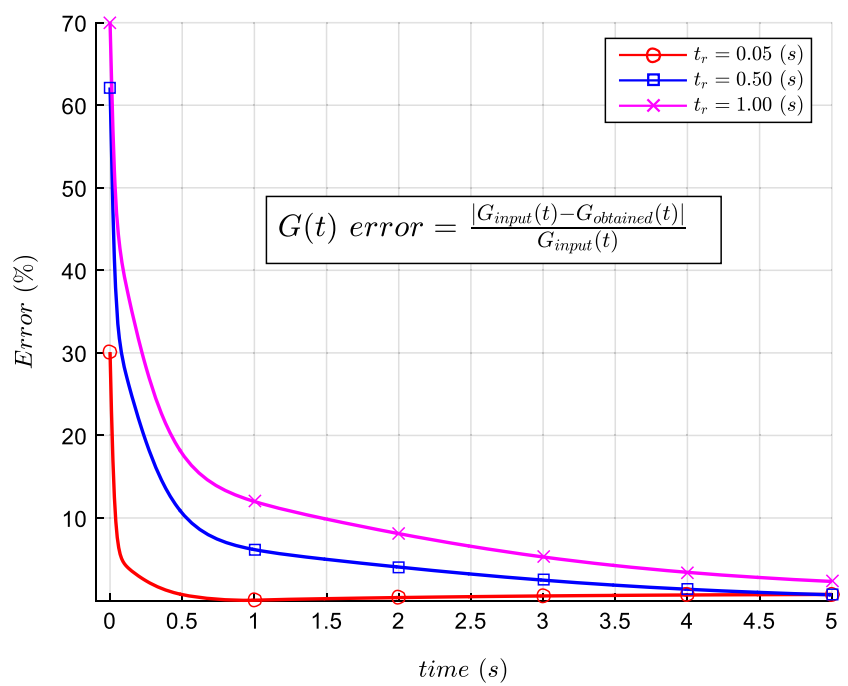

(b)

Fig. 9 The loading rate effect on the measured shear modulus (a) $G(t)$ for different loading rates (b) the error in $G(t)$ resulting from finite loading rate 
during the test, as material relaxes the compressed load frame acts as additional spring driving the indenter further into the specimen, cf. [12]. In Fig. 10, model results for $G(t)$ are reported as obtained from the model results at the presence of machine compliance. Assuming the material is incompressible, the results in Fig. 10 are given for the procedures given by equations (13) and (16). As shown in Fig. 10, neglecting the machine compliance influence on the measurements results in significant deviation of the measured $G(t)$ as compared to the input one. It has to be pointed out, that the deviation illustrated in Fig. 10, will of course depend on the magnitude of the compliance and on the viscoelasticity of the material tested. Accordingly, the deviation may be expected to be even larger for the materials with significant relaxation properties. At the same time, as shown in Fig. 10, the interpretation procedure based on equation (13), and by extension equations (7) and (8), allows to determine $G(t)$ accurately, provided that the machine compliance is taken into account.

\section{Experimental Results}

In what follows viscoelastic functions of POM and bitumenbased materials measured with instrumented indentation tests at different loading protocols are reported. Indentation test results are compared with the measurements obtained uniaxial tension and DSR tests for POM and bitumen-based specimens respectively.

The mean values of $G(t)$ for POM out of at least 10 uniaxial and indentation tests are shown in Fig. 11(a). As described in Linear Viscoelastic Characterization of POM section, the indentation relaxation test follows equation (21), while the indentation ramped test followed equation (22). The standard deviation of the results in Fig. 11(a), is shown in Fig. 11(b).

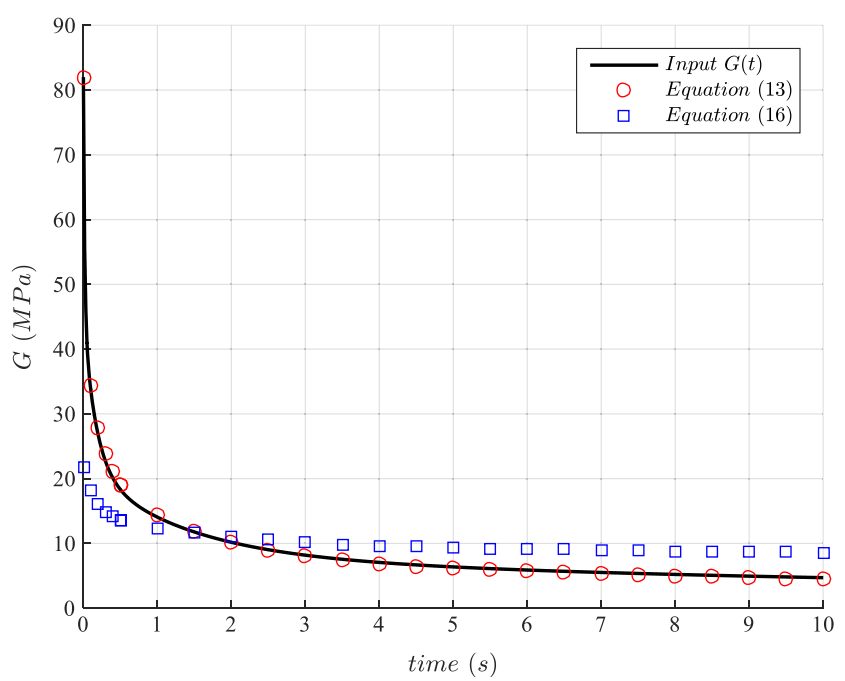

Fig. 10 Influence of machine compliance on the $G(t)$ measured at relaxation test
According to Fig. 11(a), $G(t)$ from the indentation relaxation and uniaxial testing are in reasonable agreement, the maximum deviation between the two being $12 \%$ at $t=0$, while the average differences are below $6 \%$. This is quite similar to the results reported by Huang and $\mathrm{Lu} \mathrm{[16],} \mathrm{using} \mathrm{a} \mathrm{combi-}$ nation of a spherical and Berkovich indenters and reporting a $5.9 \%$ average error, and $\mathrm{H}$. $\mathrm{Lu}$ et al. [13], reporting a $7.8 \%$ maximum error when applying a step loading function with a spherical indenter, both studies performed the measurements on polymethyl-methacrylate (PMMA). The indentation ramped test measurements exhibit, however, much larger scatter as compared to the uniaxial tests. This may be attributed to the accuracy of load measurements accuracy. $G(t)$ measured with the ramped indentation tests is approximately $80 \%$ to $13 \%$ higher as compared the other tests for $t<100 \mathrm{~s}$. The discrepancy may be attributed to the difficulty of obtaining accurate measurements at low load levels during the initial part of the ramped test, which is indirectly evidenced by much higher standard deviation of the measurements in the beginning of the test, cf. Fig. 11(b).

In measuring $\nu(t)$ only the relaxation tests results were used as it was not possible to measure $\epsilon_{\theta}(t)$ evolution with time with meaningful accuracy due to the large gauge drift in the measurement compared to the small change that occurs from ramp indentation. Accordingly, an average value of the $\epsilon_{\theta}(t)$ measured during the relaxation indentation period, has been used to estimate specimen's constant Poisson's ratio, i.e. $\nu(t)=\nu$. This is corroborated by the fact that the change in $\nu(t)$ as measured by the uniaxial creep test is small, i.e. only a maximum $4 \%$ difference from the mean value, where $\nu(t)$ changes from approximately 0.39 to 0.42 , as shown in Fig. 12. The Poisson's ratio obtained from the indentation relaxation tests is shown in Fig. 12. It is apparent that the indentation method can capture Poisson's ratio in good agreement with the uniaxial tests, with an average difference below 6\%. The corresponding average difference in $K(t)$ values is $42 \%$, obtained with the following equation in the Laplace domain:

$$
\tilde{K}=\frac{\tilde{E}}{3 *(1-2 s \times \tilde{\nu})}
$$

This larger disparity is somewhat expected, as for Poisson's ratios close to 0.5 , small changes in their values will yield large differences in corresponding $K(t)$, due to the denominator's value approaching zero.

The experimental results obtained on bitumen-based materials are reported in Figs. 13 and 14. In Fig. 13, $G(t)$ measured with the DSR relaxation test is compared with the ones obtained using relaxation and ramp-relaxation indentation tests, i.e. with the $h(t)$ defined by equations (21) and (23) respectively. As seen in Fig. 13, $G(t)$ measured in indentation ramprelaxation tests is in a reasonable agreement with the DSR 


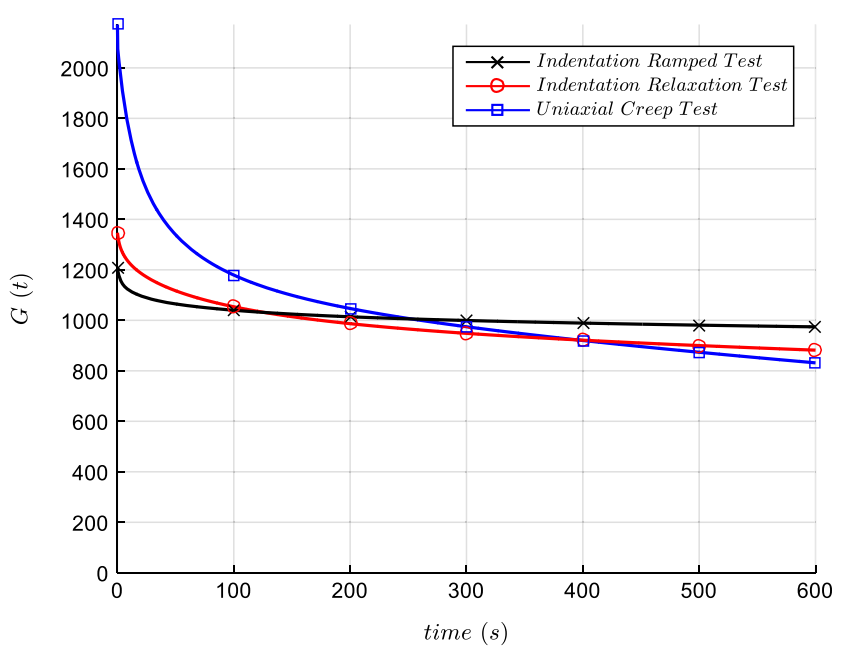

(a)

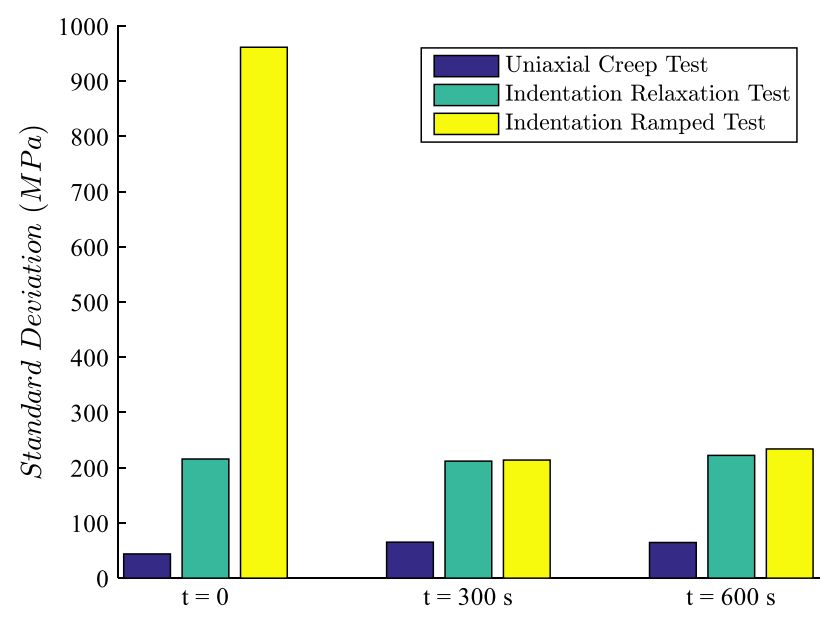

(b)

Fig. 11 (a) Averaged shear relaxation modulus comparison between different tests (POM) (b) The standard deviation at different times

results; the difference is approximately $20 \%$ at the beginning of the test and increases to $45 \%$ at $t=50 \mathrm{~s}$. The discrepancy reported in Fig. 13 can be compared to the results reported in other studies, e.g. Zofka and Nener-Plante [11] reported a $40 \%$ average difference in the bitumen's creep compliances measured with flat punch indentation and bending beam rheometer (BBR). Furthermore, according to the ASTM D7175-15 standard the acceptable scatter for the DSR measurements is $9.5 \%$ and $29.1 \%$ from the mean value for single-operator and multilaboratory precision. Accordingly a maximum deviation of $45 \%$ reported in Fig. 13 is at least comparable to the multilaboratory precision of the DSR measurements. The observed difference may be attributed to nonlinear material behaviour arising due to high localized strains under the indenter, cf. [12]. The indentation relaxation test results were analyzed

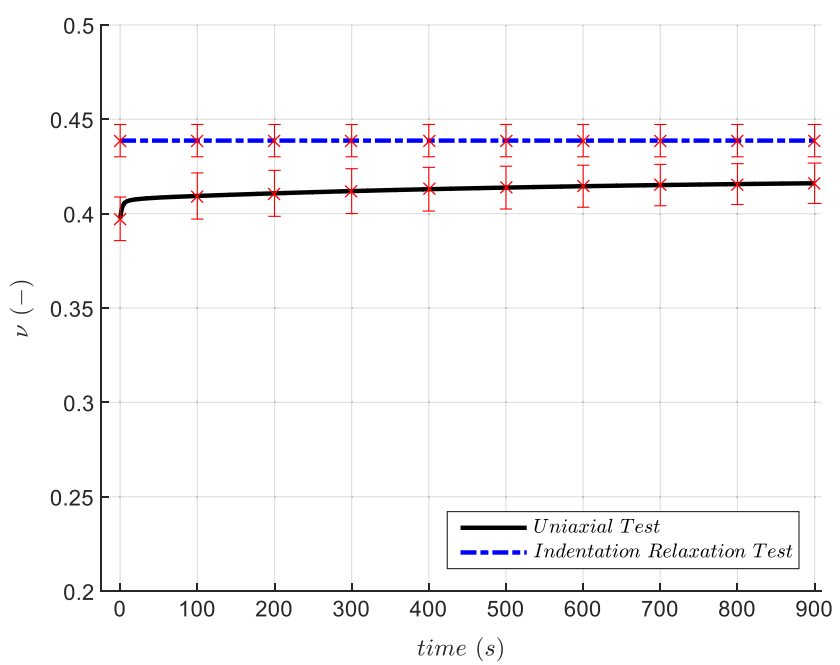

Fig. 12 Poisson's ratio obtained using uniaxial and indentation relaxation tests for POM with $95 \%$ confidence interval with analysis procedure proposed by Larsson and Carlsson [2] (equation (16)), but also with the analysis procedure based on equation (11). In the latter case, the $h(t)$ history, including finite loading rate was used, with the machine compliance taken into account. As it is clear from Fig. 13, using equation (11) significantly improves the agreement with the DSR measurements, as has been discussed above based on numerical results, cf. Fig. 10. As may also be seen from Fig. 13, ramp-relaxation tests results are in better agreement with the DSR measurements as compared with the pure relaxation tests. This may be explained by a narrower range of load magnitudes attained during the test as well as by the fact that finite loading rate is taken explicitly into account in the analysis. Accordingly, the indentation tests on bitumen-filler mastics discussed below were performed in ramp-relaxation mode.

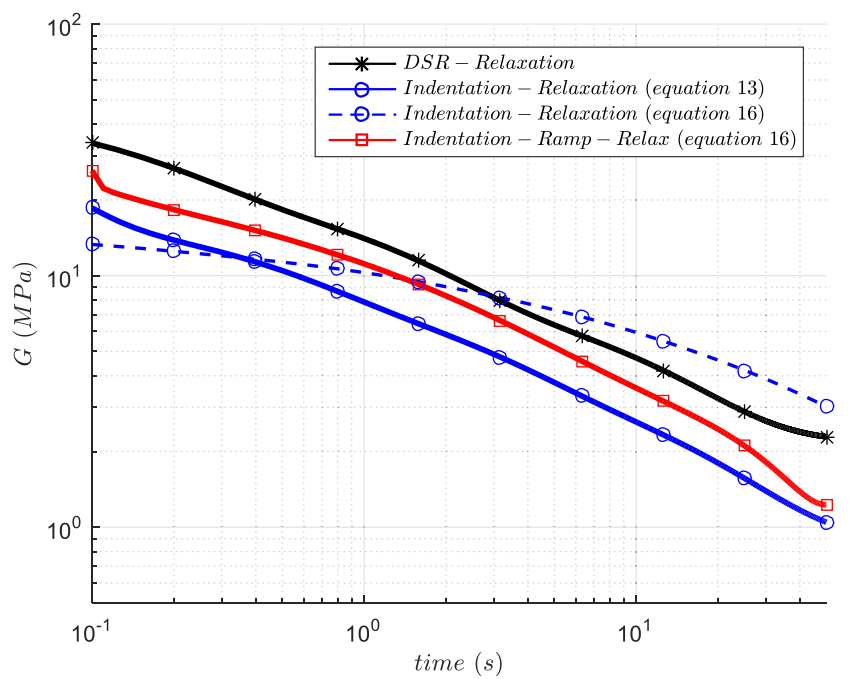

Fig. 13 Bitumen $G(t)$ measured with the DSR and indentation tests at $0{ }^{\circ} \mathrm{C}$ 


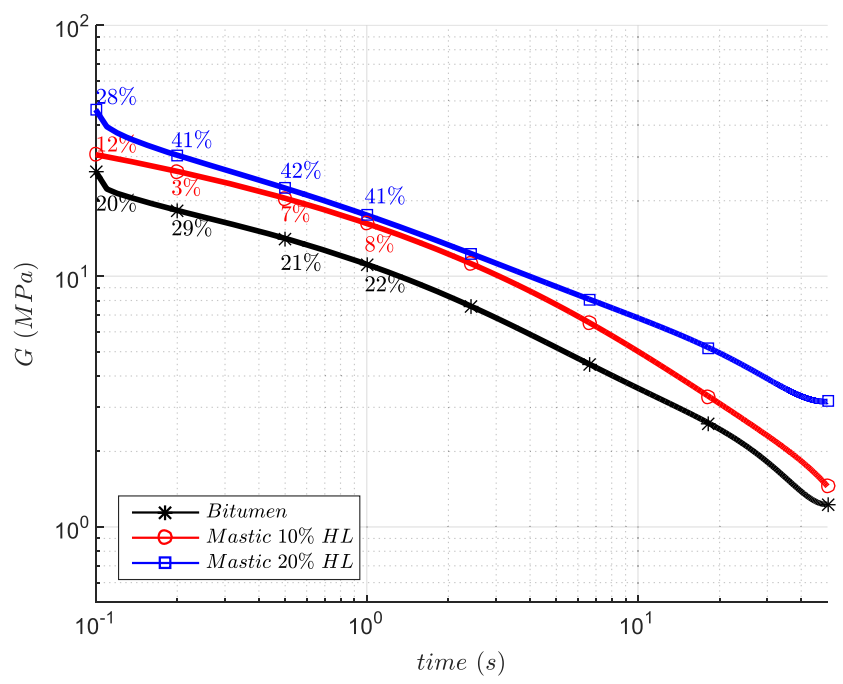

Fig. 14 Shear relaxation function measured for the different bituminous materials using ramp indentation, the numbers present the magnitude of deviation from DSR tests

Figure 14 shows $G(t)$ from indentation ramp-relaxation tests on bitumen and bitumen-filler mastics is together with the percentage of deviation from the DSR data. The DSR measurements on bitumen-filler mastics have been performed in frequency sweep mode and converted to $\mathrm{G}(\mathrm{t})$ for $t<1 \mathrm{~s}$. As may be seen addition of the hydrated lime resulted in increasing $G(t)$. The average increase in $G(t=0.1 \mathrm{~s})$ measured in the DSR was $6 \%$ and $96 \%$ for the $10 \%$ and $20 \%$ filler respectively. This can be compared to the corresponding values of $17 \%$ and $76 \%$ obtained with the indentation tests. The increase in the measured $G(t=0.1 \mathrm{~s})$, using the indentation technique, is also reasonably close to the stiffening effect of $17 \%$ and $79 \%$ as predicted by DSR measurements of Buttlar et al. [24]; their DSR measurements were the average of results obtained at temperatures $-15{ }^{\circ} \mathrm{C}, 25{ }^{\circ} \mathrm{C}$ and $70{ }^{\circ} \mathrm{C}$ from which Buttlar et al. [24] concluded that the temperature has a minimal effect on the stiffening due to filler addition. As seen in Fig. 14, the difference between the DSR and indentation tests is within $30 \%$ for bitumen and $10 \%$ bitumen-filler mastics and is approximately $40 \%$ for the mastic with $20 \%$ filler. This, again is comparable with the almost $30 \%$ acceptable scatter for the multi-laboratory DSR measurements on bitumen, as discussed above in connection to Fig. 13. The variations in shapes of the $G(t)$ curves measured with indentation tests for bitumen and bitumen-filler mastics are also attributed to the tests variability; the average coefficients of variation are $33 \%$ for bitumen and $30 \%$ and $91 \%$ for $10 \%$ and $20 \%$ bitumen-filler mastics respectively. The $G(t)$ measured at indentation tests is furthermore consistently lower as compared to the DSR results for all the loading procedures and materials investigated. This indicates that non-linear material behavior, such as power-law creep cf. Ossa et al. [25], may be a possible explanation for the observed quantitative discrepancies.

\section{Conclusions}

An analysis method allowing to measure two independent viscoelastic functions with the spherical indentation test at arbitrary non-decreasing loading history has been proposed in the present study and verified with numerical results. Moreover, a computational study on the test sensitivity to the main measurement parameters has been performed. It has been shown numerically that provided that specimens dimensions are not extremely small, both $G(t)$ and $v(t)$ functions can be measured accurately with the spherical indentation. The influence of errors in measured $h(t)$ and $\epsilon_{\theta}(t)$ on the determined viscoelastic functions has been quantified. It is furthermore shown that the developed method allows accounting for the influence of finite loading rate as well as of the machine compliance on the measurements, this provides as an improvement on the method proposed in [2] which is strictly applicable only when loading is defined by the Heaviside step function.

The experimental investigations on POM have shown, that the indentation test can be used to measure both $G(t)$ and $\nu$ with a reasonable accuracy. The average difference between the viscoelastic parameters measured with the uniaxial and relaxation indentation tests were found to be $6 \%$. In case of ramp loading experiments, however, the developed method, is very sensitive to errors in load measurements.

Shear relaxation moduli of bitumen and bitumen-filler mastics have been measured with the DSR and instrumented indentation tests. Performing a perfect relaxation test on bitumen was found to be practically challenging due to machine compliance effects Provided that the machine compliance is known, the analysis procedure proposed presently allows accounting for its effect on the measurements. An optimal loading scheme for the materials with profound relaxation properties has been proposed, i.e. ramp-relaxation indentation test.; which allows to reduce the range of loads attained during the test. The relaxation moduli measured with the indentation tests on all tested bitumen-based materials were found to be consistently lower than the corresponding values obtained with the DSR, the maximum difference was found to be $40 \%$ for the mastics with $20 \%$ filler. One possible explanation for the observed quantitative discrepancy is the non-linear material behavior in the vicinity of the contact area. Furthermore, similarly to the results reported previously in the literature, it has been observed that addition of the filler resulted in material stiffening. The stiffening effects of the filler measured with the indentation tests were found to compare favorably with the DSR results.

Acknowledgements Open access funding provided by Max Planck Society. The writers are indebted to Mr. M. Öberg and Ms. I. L. Arregui for the very helpful experimental assistance. Authors would also like to thank Dr. X. Lu and Mr. P. Uhlback, Nynas AB, for providing the materials and performing the DSR testing and Prof. Manfred Partl for his valuable comments on the manuscript. 
Open Access This article is distributed under the terms of the Creative Commons Attribution 4.0 International License (http:// creativecommons.org/licenses/by/4.0/), which permits unrestricted use, distribution, and reproduction in any medium, provided you give appropriate credit to the original author(s) and the source, provide a link to the Creative Commons license, and indicate if changes were made.

\section{References}

1. Oliver WC, Pharr GM (2004) Measurement of hardness and elastic modulus by instrumented indentation: Advances in understanding and refinements to methodology. J Mater Res 19(1):3-20

2. Larsson P-L, Carlsson S (1998) On microindentation of viscoelastic polymers. Polym Test 17(1):49-75

3. Alcalá J, Giannakopoulos AE, Suresh S (1998) Continuous measurements of load-penetration curves with spherical microindenters and the estimation of mechanical properties. J Mater Res 13(5): $1390-1400$

4. Venkatesh TA, Van Vliet KJ, Giannakopoulos AE, Suresh S (2000) Determination of elasto-plastic properties by instrumented sharp indentation: guidelines for property extraction. Scr Mater 42(10): 833-839

5. Storåkers B, Biwa S, Larsson P-L (1997) Similarity analysis of inelastic contact. Int J Solids Struct 34(24):3061-3083

6. Bucaille JL, Felder E, Hochstetter G (2002) Identification of the viscoplastic behavior of a polycarbonate based on experiments and numerical modeling of the nano-indentation test. J Mater Sci 37(18):3999-4011

7. Raghavan A, Gu D, Nguyen X, VanLandingham T, Karim M, Raghavan D, Gu X, Nguyen T, VanLandingham M, Karim A (2000) Mapping polymer heterogeneity using atomic force microscopy phase imaging and nanoscale indentation. Macromolecules 33(7):2573-2583

8. Jäger A, Lackner R, Stangl K (2007) Microscale characterization of bitumen - back-analysis of viscoelastic properties by means of nanoindentation. Int J Mater Res 98(5):404-413

9. Tarefder RA, Zaman A (2011) Characterization of asphalt materials for moisture damage using atomic force microscopy and nanoindentation. In: Gopalakrishnan K, Birgisson B, Taylor P, Attoh-
Okine NO (eds) Nanotechnology in Civil Infrastructure. Springer Berlin, Heidelberg, pp 237-256

10. Sirghi L, Rossi F (2006) Adhesion and elasticity in nanoscale indentation. Appl Phys Lett 89(24):243118

11. Zofka A, Nener-Plante D (2011) Determination of asphalt binder creep compliance using depth-sensing indentation. Exp Mech 51(8):1365-1377

12. Jelagin D, Larsson P-L (2013) Measurement of the viscoelastic properties of bitumen using instrumented spherical indentation. Exp Mech 53(7):1233-1244

13. Lu H, Wang B, Ma J, Huang G, Viswanathan H (2003) Measurement of creep compliance of solid polymers by nanoindentation. Mech Time-Dependent Mater 7(3):189-207

14. Lee EH, Radok JRM (1960) The contact problem for visooelastic bodies. J Appl Mech 27(3):438-444

15. Oyen ML (2006) Analytical techniques for indentation of viscoelastic materials. Philos Mag 86(33-35):5625-5641

16. Huang $\mathrm{G}, \mathrm{Lu} \mathrm{H}$ (2006) measurements of two independent viscoelastic functions by nanoindentation. Exp Mech 47(1):87-98

17. Hill R, Storakers B, Zdunek AB (1989) A theoretical study of the brinell hardness test. Proc R Soc London A Math Phys Eng Sci 423(1865):301-330

18. Hertz H (1882) Ueber die Berührung fester elastischer Körper. J Reine Angew Math 1882(92):156-171

19. Johnson KL (1985) Contact mechanics. Cambridge University Press, Cambridge

20. VanLandingham MR, Chang N-K, Drzal PL, White CC, Chang S$\mathrm{H}$ (2005) Viscoelastic characterization of polymers using instrumented indentation. I. Quasi-static testing*. J Polym Sci Part B Polym Phys 43(14):1794-1811

21. Lu X, Langton M, Olofsson P, Redelius P (2005) Wax morphology in bitumen. J Mater Sci 40(8):1893-1900

22. Starkova O, Aniskevich A (2007) Limits of linear viscoelastic behavior of polymers. Mech Time-Dependent Mater 11(2):111-126

23. Mun S, Chehab GR, Kim YR (2007) Determination of Timedomain Viscoelastic Functions using Optimized Interconversion Techniques. Road Mater Pavement Des 8:351-365

24. Buttlar WG, Bozkurt D, Al-Khateeb GG, Waldhoff AS (1999) Understanding asphalt mastic behavior through micromechanics. J Transp Res Board TRR 1681(99):157-169

25. Ossa EA, Deshpande VS, Cebon D (2005) Spherical indentation behaviour of bitumen. Acta Mater 53(11):3103-3113 